\title{
The Innate Immune System and Aging: What is the Contribution to Immunosenescence?
}

\author{
T. Fulop ${ }^{1, *}$, C. Fortin ${ }^{1}$, O. Lesur ${ }^{2}$, G. Dupuis ${ }^{3}$, J. R. Kotb ${ }^{4}$, J. M. Lord ${ }^{5}$ and A. Larbi ${ }^{6}$
}

${ }^{1}$ Research Center on Aging, Department of Medicine, Immunology Postgraduate Programme, Faculty of Medicine and Health Sciences, Université de Sherbrooke, Sherbrooke, Qc, Canada

${ }^{2}$ Etienne Lebel Research Center and Divisions of Pneumology \& Intensive Care Medicine, Department of Medicine, Faculty of Medicine and Health Sciences, Université de Sherbrooke, Sherbrooke, Qc, Canada

${ }^{3}$ Department of Biochemistry, Immunology Postgraduate Programme, Faculty of Medicine and Health Sciences, Université de Sherbrooke, Sherbrooke, Qc, Canada

${ }^{4}$ Department of Medicine, Division of Haematology, Faculty of Medicine and Health Sciences, Université de Sherbrooke, Sherbrooke, Qc, Canada

${ }^{5}$ MRC Center for Immune Regulation, School of Immunity and Infection, University of Birmingham, Birmingham, UK

${ }^{6}$ Singapore Immunology Network (SIgN), Biopolis, A*STAR, Singapore

\begin{abstract}
The immune system plays an important role in the defence against various threats to health, such as pathogens, cancer cells or modified-self proteins. With aging there is a decrease in the immune response, called immunosenescence, concomitantly with the increase in some age-related diseases such as infections, autoimmune disorders, chronic inflammatory diseases and cancer. The immune response is traditionally divided between innate and adaptive immune responses. Accumulating evidence suggests that immunosenescence is not only restricted to the adaptive but also affects the innate immune system. Assessment of innate immune system functions revealed that it is also susceptible to age-related dysregulation. Furthermore, it is becoming clear that the sustained function of innate cells is indispensable for the adequate functioning of the adaptive immune response. This review will describe the changes in the innate immune response with aging and the recent discoveries, which may shed new light on its contribution to immunosenescence.
\end{abstract}

Keywords: Aging, immunosenescence, innate immunity.

\section{INTRODUCTION}

The immune system plays an important role in the defence against various invaders, such as pathogens, cancer cells or modified-self proteins. With aging there is a decrease in the immune response, called immunosenescence, concomitantly with the increase in some age-related diseases such as infections, autoimmune disorders, chronic inflammatory diseases and cancer [1-3]. Whether a relationship between these diseases and the immune alterations exists is still poorly understood. However, experimental evidence supports the proposal that an association may exist between immunosenescence and the higher incidence of infections with aging $[3,4]$.

The immune response is traditionally divided between innate and adaptive immune responses, though it is clear that communication between the two is essential for a complete response to pathogens [5]. The attention of immunologists

*Address correspondence to this author at the Research Center on Aging, University of Sherbrooke, 1036, rue Belvedere sud, Sherbrooke, Qc, J1H 4C4, Canada; Tel: +1 819829 7131; Fax: +1 819829 7141;

E-mail: tamas.fulop@usherbrooke.ca has focused on T lymphocyte dysregulation with age as their alterations were considered as the hallmark of immunosenescence $[3,6]$. Accumulating evidence now suggests that immunosenescence is not only restricted to the adaptive but also affects the innate immune system $[7,8]$. Furthermore, it is clear that the sustained function of innate cells is indispensable for the adequate functioning of the adaptive immune response [9]. Thus, this review will describe the changes in the innate immune response with aging and the recent discoveries, which may shed new light on its impact on immunosenescence.

\section{HOW AGING AFFECTS NEUTROPHILS}

Neutrophils are the first cells to arrive at the site of inflammation caused either by an infection or tissue damage [10]. Tissue resident activated macrophages contribute to their recruitment via the secretion of cytokines such as TNF$\alpha$ and IL-1 and chemokines such as IL-8 [11]. Their ability to be recruited by chemotaxis is one of their important functions [12]. Once at the inflamed site, neutrophils are able to engulf the invaders by phagocytosis and destroy them intracellularly by the generation of reactive oxygen and ni- 
trogen species and release of proteolytic enzymes from cytoplasmic granules. In some circumstances they are also able to kill them extracellularly, for example by the extrusion of DNA coated with antimicrobial agents, termed neutrophil extracellular traps [13]. Upon completion of their task neutrophils undergo a programmed cell death (apoptosis) [12]. At the molecular level, signalling pathways including JakSTAT, PI3K and the MAPKs were identified as important regulators of neutrophil functions (Fig. 1).

Several functions of neutrophils are altered during human aging though it remains to be established whether there is a global reduction at the functional level $[14,15]$. To date several functions are reduced with aging, including phagocytosis, chemotaxis, and intracellular killing via free radical production [15-17]. Homeostasis of neutrophils is also changed as their susceptibility to spontaneous and induced apoptosis is increased in aged individuals [18]. The functional changes observed to date suggest that neutrophil functions are severely compromised with aging. The question arising is why this occurs.

\subsection{Neutrophil Receptor Signalling Pathways in Aging}

As for all other cells in the organism neutrophils have several very important receptors mediating their various functions. Historically fMLP, Fc $\gamma$ and the C3b receptors were extensively studied. It was shown that the functions mediated by these receptors are altered with aging and it was found that changes in their signalling pathways were the causes of these functional alterations [19]. The most affected pathways were the MAP kinases, the Jak/STAT and the PI3K-Akt pathways [20]. The changes in these pathways are not due to the changes in the number of receptors but to the changes in the membrane composition including that of membrane microdomains named lipid rafts. The decline in intracellular signalling pathways contributes to the agerelated impairment in neutrophil functions [21].

Recently, one of the most important discoveries was the description of pattern recognition receptors (PRR) on the surface of many immune cells including neutrophils recognizing pathogen associated molecular patterns (PAMPs) [22]. The ever growing family of the PRRs now includes three main types: the Toll like receptors (TLRs), retinoic acid inducible gene 1 protein (RIG-1)-like helicases (RLRs) and nucleotide binding domain and leucine-rich-repeatcontaining proteins (NLRs) [23]. It is now fully recognized that they play an essential role in many cell functions, including neutrophil biology. They can allow immune cells to discriminate between self and non-self. They also act as danger sensing receptors as they alert the organism that an aggression from microorganisms, transformed cells or damaged cells.

There are currently 13 TLRs described with various recognition specificities and signalling pathways leading to well characterized cellular responses [24]. TLR2 and TLR4 recognize mainly bacterial products and their signal transduction is mediated by the MyD88 pathway [25] and also by the recently described TRIF pathway [26,27]. Pathogens can also be recognized intracellularly, for example viral products

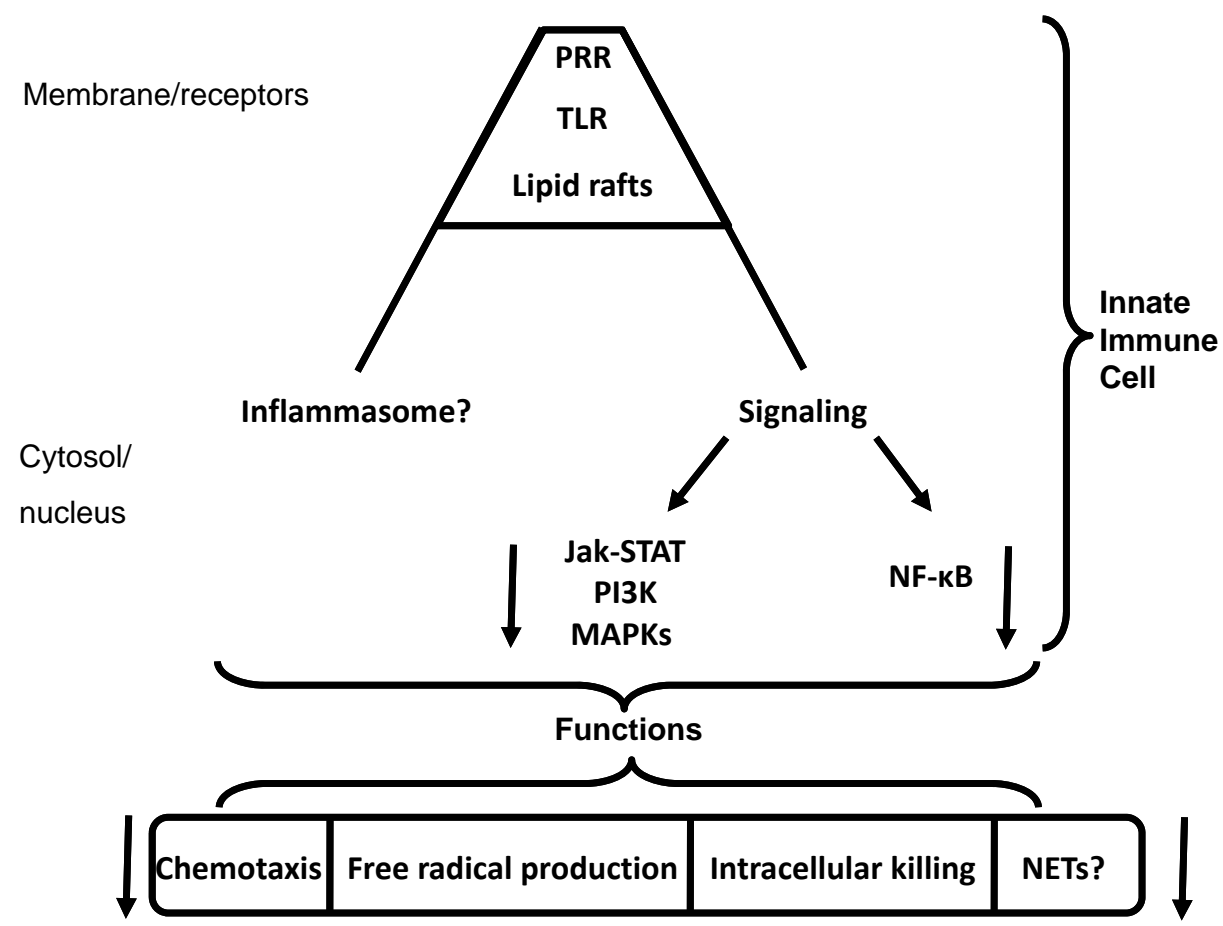

Fig. (1). Cause and consequence of altered signaling in innate immune cells during aging. The pattern recognition receptors (PRR) on the surface of many immune cells include the Toll like receptors (TLRs). The dysregulation of PPR and TLR expression or localization within membrane lipid rafts during aging will have several consequences. The altered cellular functions are chemotaxis, free radical production and intracellular killing. The study of the mechanisms to explain such dysfunction revealed that the Jak-STAT, PI3K and MAPKinases pathway dysregulation were involved altogether leading to an altered NK-kB translocation. Whether other intracellular processes such as inflammasome functionality and their impact of functions such as NETs (Neutrophil Extracellular Traps) formation are also altered with age is still unknown. $\downarrow$ : decrease with aging. 
such as RNA or DNA products are recognised via TLR3 and TLR7. Their signalling is mediated by the TRIFF pathway. The activation of these TLRs results in the activation of NF$\kappa \mathrm{B}$, a transcription factor yielding a high production of cytokines [28]. Neutrophils from aged individuals display alterations in the signalling of these TLRs leading to altered functions of neutrophils $[14,29]$. While the number of these receptors is not significantly changed with aging there is a significant alteration in the trafficking of signalling molecules in and out of lipid rafts (Fig. 1).

The retinoic acid inducible gene 1 protein (RIG-1)-like helicases (RLRs) and nucleotide binding domain and leucine-rich-repeat-containing proteins (NLRs) have recently been identified as members of the PRR network [30,31]. They are able to modulate various responses to viruses. The response can be a pro-inflammatory response or a direct antiviral response but both types are mediated by the inflammasome [23]. The inflammasome is a complex of molecules activated by specific PRRs (NLRs and AIM2) and responds specifically to an aggression via the activation of inflammatory caspases such as caspase- 1 and caspase-5, resulting ultimately in the production of a wide range of cytokines [32]. There are currently no data on how these inflammasomes are affected by aging and. We can suggest after the alterations in neutrophil functions that their assembly and function may be altered. This important area needs further study.

Considering the collected data we can conclude that the functionality of PRRs is altered with aging further contributing to the functional alteration of neutrophils [29].

\subsection{Neutrophil Extracellular Activity with Aging}

It is now recognized that structures called neutrophil extracellular traps (NETs) are released by neutrophils which are capable of mediating various functions [13]. This seems to be an alternative to neutrophil death driven by necrosis or apoptosis. During NETosis the nuclei swell and the chromatin is dissolved. This decondensed DNA carries with it proteins from the cytosolic granules. Most of these associated proteins are histones, defensins, elastase, lactoferrin which provide antimicrobial functions [33]. The NETs are capable of binding to both Gram negative and Gram positive bacteria. The exact process of their formation is not yet fully elucidated although emerging data suggests that PAD4 as well as the NADPH oxidase seem to play a central role [34].

Whether the functionality of NETs is sustained with aging is not known. However as they are mostly proinflammatory it is possible that their activity is increased and may contribute to the pro-inflammatory status associated with aging, termed Inflamm-aging. Moreover, NET production is altered in several pathologies such as SLE with a deficient DNase-1 activity, which could also occur with aging [35]. In contrast, as they may also have a role in the containment of infections and inflammations, their production could be decreased with aging helping to maintain the inflammatory process. Further research is clearly required to establish the effect of aging upon this important function.

\subsection{Neutrophil Subpopulation Changes with Aging}

For a long time it was not fully recognised that neutrophils could be present as subpopulations, but recently it has

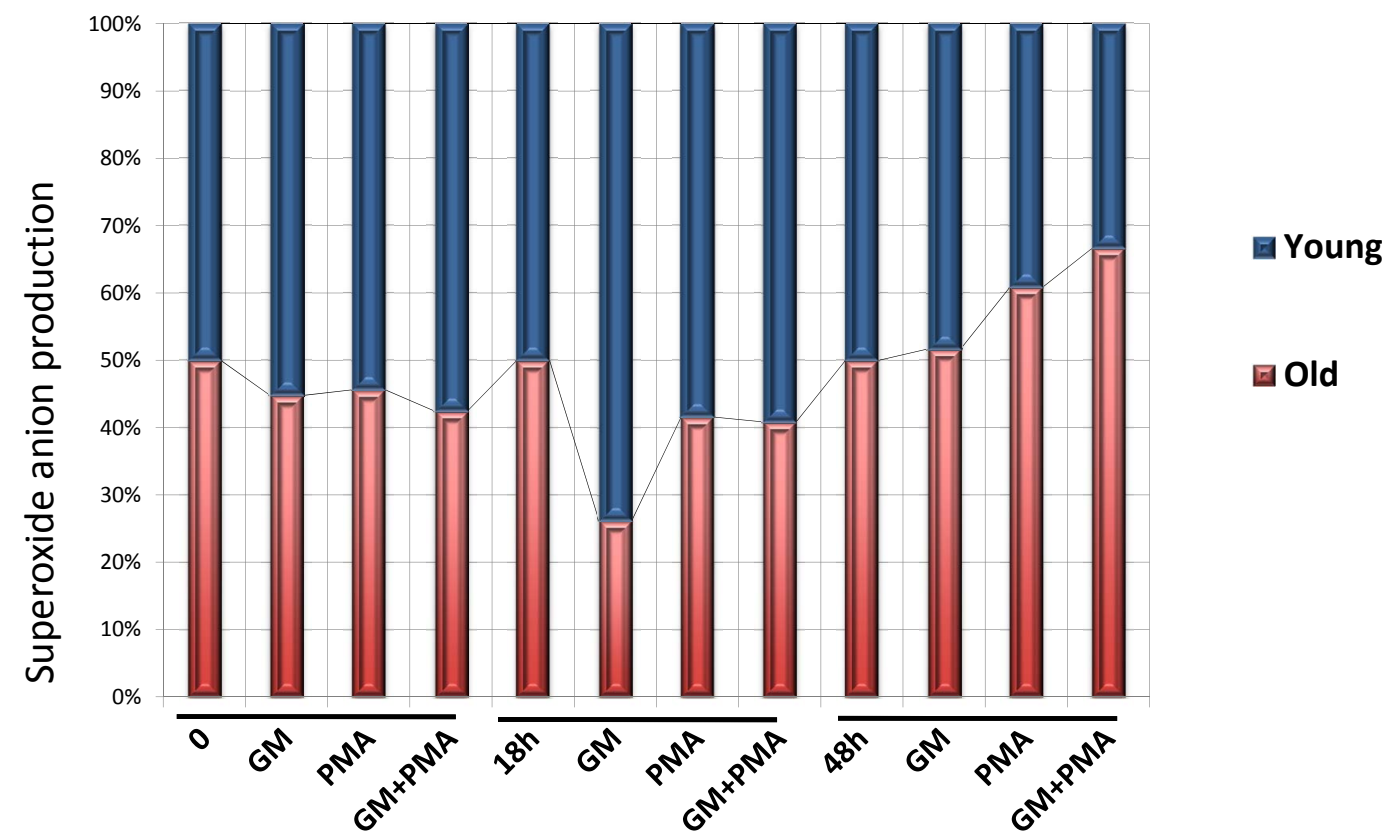

Fig. (2). Higher functionality of long-lived neutrophils in elderly individuals. Neutrophils were isolated and stimulated with GM-CSF (GM), PMA or both immediately, 18 hours and 48 hours after isolation. Production of superoxide anion was recorded using the cytochrome c assay. The aim of this experiment is to show the existence of long-lived neutrophils in aged individuals (at 48 hours). Most of neutrophils undergo apoptosis at 18 hours unless stimulated with the anti-apoptotic GM-CSF and altered response to GM-CSF in the elderly may explain reduced functionality. However, later in the course of the experiment, neutrophils that survived due to GM-CSF stimulation display similar or even higher response to re-stimulation than that of younger individuals, suggesting the existence of such "super long-lived functionally intact neutrophils" 
revealed that neutrophils are not a homogenous cell population [36]. Sophisticated techniques helped to show that subpopulations exist in various stages from dormant to primed to fully activated. With aging we have hypothesized several years ago that neutrophils are not a homogeneous cell population, but exist in distinct subpopulations to regulate the timing, duration, intensity and type of the inflammatory response. We have shown that after 48 and 72 hours in vitro cell culture there was an apoptosis-resistant neutrophil subpopulation even in elderly subjects with a relatively well preserved functionality (Fig. 2) while most of the neutrophils had undergone apoptosis [Fulop et al, unpublished observations]. At that time we were not able to further characterize them, but we are intensively trying to characterize them phenotypically and functionally at the present time.

In summary, the published data strongly support the hypothesis that dysfunction of neutrophils with aging contributes to immunosenescence and the development of various age-related diseases, as will be described later in this review.

\section{HOW AGING AFFECTS MONOCYTE/MACRO PHAGES AND DENDRITIC CELLS}

The monocyte, macrophage, dendritic cell system is the other fundamental cellular part of the innate immune response [37]. These cells play essential functions on one hand against invaders such as viruses, bacteria and transformed cells and on the other hand are the link to the adaptive immune system via their role in antigen presentation [38,39]. Monocytes are precursors of macrophages and dendritic cells. Macrophages are in the first line of defense against invading pathogens in tissues $[22,40]$ and there is a polarization of macrophages reflecting their functional heterogeneity. Macrophages can be classified as classically activated (M1) or alternatively activated (M2). The M1/M2 polarization may reflect the bidirectional macrophage-lymphocyte interaction, namely Th1 $\mathrm{T}$ cells drive M1 polarization as via IFN $\gamma$, while Th2 cells induce M2 polarization via IL-4 and IL-13 [9].

It is clear that neutrophils contribute to the recruitment and activation of macrophages by generating chemotactic factors, such as MIP- $1 \alpha$ and MIP- $1 \beta$ as well as IL-8 $[41,42]$. Neutrophils can also influence the differentiation of macrophages into pro-inflammatory and anti-inflammatory subpopulations [41,43]. Finally, release of IFN- $\gamma$ may also activate macrophages [44]. There is a very active interaction between neutrophils and macrophages at the site of inflammation as neutrophils activate macrophages and macrophages contribute to the survival of neutrophils via production of pro-survival cytokines.

\subsection{Monocyte/Macrophages Functional Changes with Aging}

In rodents many data exist showing macrophage functional changes with aging, however much less is known regarding changes in humans $[3,7,8,45,46]$. Nevertheless, data suggest that monocyte/macrophages from aged individuals display age-related dysfunction [47-49]. These alterations include a decrease of cell surface TLRs expression (TLR1 and TLR4) though this finding has not been unequivocally demonstrated [3,7,48,50]. In addition TLR signalling pathways show age-related alterations [29] and this has been linked to altered chemotaxis as shown by the reduced number of infiltrating macrophages in wounds of elderly humans [51,52]. There is also a decrease in expression of CD80/CD86 co-stimulatory receptors on monocytes upon TLR stimulation which may impact on the seroconversion efficiency following influenza vaccination [53]. In vitro studies in humans demonstrated a higher pro-inflammatory cytokine profile, especially for IL-6 and IL-8 in resting monocytes [54], though cytokines production upon stimulation with LPS is reduced. Macrophages from elderly produce more prostaglandin E2 which drives suppression of the T cell activation via decreased IL-12 production [55]. Furthermore, it was demonstrated that the phagocytosis and free radical production were reduced in monocytes/macrophages with healthy aging [56].

Recently, it has been shown that young patients infected with West Nile virus show reduced TLR3 expression on the surface of macrophages, while in elderly there was a dysregulation and a persistent TLR3 expression [57]. This may lead to an elevated inflammatory response causing an excess morbidity of elderly suffering from infection. Thus, the age-related altered TLR responses may contribute in some circumstances to the increased risk of infectious diseases with aging and subsequent increased frailty in the elderly.

Together experimental data suggest that with aging most of the monocyte/macrophage functions are changed with aging leading to altered pathogen clearing, regulation of the adaptive immune response and the inflammatory process resulting in chronic inflammation and increased age-related diseases such as infections, cardiovascular disease and cancers.

\subsection{Dendritic Cell Functional Changes with Aging}

DCs are the most potent antigen presenting cells that can prime specific $\mathrm{T}$ cells. There are several types of dendritic cells (DCs) [58]. The plasmocytoid dendritic cells (pDCs) are important in host defense against viral infections as they are one of the first cells to produce type I interferon and as such they may initiate several other responses, including NK cell activation which will amplify the host response and help to clear the virus [59-61]. As already mentioned, in this function the TLRs play a fundamental role as TLR7/9 are able to interact with the virus components inside of the endosomes. The signalling events elicited by these endosome residing TLRs will lead via the interferon regulatory factor to the increased secretion of type I interferon. The second type of dendritic cell is the conventional or myeloid dendritic cells (mDCs). They are the most important antigen presenting cells to $\mathrm{T}$ lymphocytes. They express TLRs and C-type lectins for the detection of antigens, including viruses. They produce IL-12, IL-15 and IL-18. IL-12 is essential to induce Th1 cell responses which will induce cytotoxic $\mathrm{T}$ lymphocyte responses to clear virus infected cells [28]. They can also activate NK cells. Besides presenting the antigens they also provide co-stimulatory signals and cytokines for optimal 
$\mathrm{T}$ cell priming resulting in differentiation and proliferation of $\mathrm{T}$ cells [62].

There are several studies demonstrating alterations in pDC function in aged humans including reduced type I interferon production via the TLR stimulation e.g. TLR7 and TLR9 [28]. Several virus derived molecules such ssRNA or CpG were unable to elicit TLR7 or TLR9 activation [63,64]. It seems that the increased basal oxidative stress related to aging could be the underlying cause of the decreased upregulation of the interferon regulatory factors by TLRs $[16,65]$. There is still controversy around whether the TLRs response to stimulation is altered with aging in macrophages or mDCs. In the mean time they seem to retain the capacity to produce pro-inflammatory cytokines and to activate CD8+ $\mathrm{T}$ cells [66] as well as the induction of IL-17 which is known to recruit neutrophils [67]. Thus, an exaggerated DCs response may alter the otherwise beneficial response to viral infections in elderly through a putative exaggerated proinflammatory response [68].

In the case of influenza infection which is known to be devastating in elderly because of the decreased immune response, more data are emerging suggesting that the increased susceptibility of the elderly is due in large part to the alterations in the innate immune response $[23,61,68,69]$. More specifically the TLR responses in DCs may be predictive of the efficacy of the influenza vaccine. In response to various TLR stimulations such as TLR1/2, the production of various cytokines including TNF, IL-6, IL-12 p40 is altered [70]. Changes in the expression of these TLRs were described extracellularly and intracellularly [29]. However, these proinflammatory cytokines were found to be increased upon influenza infection in elderly suggesting that these proinflammatory cytokines are either generated from other cell types or independently of the TLRs receptors [63].

Recently, the role of the NLRP3 inflammasome has proved to be an essential component of the host defense against influenza infection, in mice, by its capacity to autoactivate caspase-1 [31]. Activated caspase-1 cleaves inactive cytokines precursors to a secreted and biologically active form such as IL-1 $\beta$, IL-18, IL-33 and IL-17. These proinflammatory cytokines further recruit monocytes and neutrophils into the lung to control influenza infection and pathogenesis [71]. Moreover, activation of CD4+ T cells is also mediated by this complex. No information exists on the functioning of the inflammasome with aging in humans, however a decrease in complex assembly and reduced proinflammatory cytokine production, was shown in mice lacking this complex. Thus, the alteration of the innate immune response to influenza infection is largely contributing to the pathogenesis of influenza virus besides the well-known role of the adaptive immune response [22].

DC appear to be altered not only in their basic functions such as phagocytosis, chemotaxis and production of IL-12, but also in their ability to activate naïve CD4+ T cells via antigen presentation $[63,72]$ and this has been attributed to decreased PI3K activity [73]. Reduced PI3K was implicated in both age-related reduced DC migration and also as a negative regulator of TLR signalling. DCs have reduced antigen processing capacity concomitantly with the altered expres- sion and function of their co-stimulatory molecules. Finally, DCs from the elderly contribute to the constant proinflammatory status observed with aging by the increased IL-6 and TNF- $\alpha$ production in their basal state [63].

\section{HOW AGING AFFECTS NK CELLS}

Natural killer (NK) cells play an important role in early pathogen defense especially against viral infections before the adaptive immune response becomes fully functional $[8,74,75]$. They also participate in the antitumoral response by producing pro-inflammatory cytokines and destroying the tumor cells [76]. NK cells produce IFN- $\gamma$ which in turn activates macrophages to kill phagocytosed microorganisms. NK cells also have innate immunoregulatory effects on adaptive responses by modulating its magnitude and quality $[76,77]$. NK cells are now recognized as a heterogeneous population classically divided into two functional subsets based on their cell surface density of CD56, CD56 bright immunoregulatory cells and CD56 ${ }^{\mathrm{dim}}$ cytotoxic cells [78]. CD56 ${ }^{\mathrm{dim}}$ cells can be further divided between licenced or unlicensed NK cells by self MHC class I [79]. Furthermore, they present activating and inhibitory surface receptors determining their overall functionality [80].

\subsection{NK Cell Functional Changes with Aging}

Studies in very healthy elderly populations revealed that the NK cell number tends to increase while the cytotoxicity is not significantly affected [81-83] and this is contributing to longevity and successful aging $[84,85]$. Other studies in unselected elderly populations showed decreased NK cell functions with aging which was associated with higher incidence of infectious diseases [86,87]. The IL-2 induced NK cell proliferation is decreased with aging [81]. Many cytokines and chemokines produced by NK cells are also decreased with aging, such as IL-2, IL-8 [88], while that of IFN- $\gamma$ seems to be maintained [84]. This decreased production of cytokines contributes to the altered activation of macrophages with aging resulting in decreased microbicidal and tumoricidal activities. Thus, NK cells of elderly show decreased proliferative response to cytokines, higher cytotoxic capacity under certain cytokine stimulations including IL-2, IL-12 or IFN $\gamma$ and a higher sensitivity to stimulation via CD16 $[89,90]$. The cytotoxic activity of NK cells with aging is depending whether the whole population of NK cells is considered or on per cell basis. As the number of NK cells is increasing and CD16 medicated ADCC does not change overall the cytotoxicity is not affected or even increased. However, on a per cell basis is decreased.

Recently, another receptor in relation to the cytotoxic activity of NK cells has been shown to be decreased with aging, namely NKp30 and NKp46, members of natural cytotoxicity receptors $[84,85]$. NKp30 has been also shown to be important in the regulation of the cross-talk between NK cells and DCs. The ligation of this receptor by an unknown ligand on DCs can lead either to a direct killing of DCs by NK cells or to the secretion of IFN- $\gamma$ and TNF- $\alpha$ and the subsequent maturation of DCs [90]. By this interaction the NK cells can activate the DCs to more efficiently prime T cells. In the meantime DCS release Th1 cytokines which further 
enhance NK activation [91,92]. Thus, NK cells via this interaction with DCs can modulate the adaptive immune response against virus-infected or tumor cells [74].

\subsection{NK Cell Phenotype and Subpopulation Changes with Aging}

The total number of NK cells is increasing with aging [93] which could be a compensatory mechanism in the aging immune system [94] however the subset distribution is changed with aging as the number of NK cells with CD56 ${ }^{\mathrm{dim}}$ increases, while those with CD56 ${ }^{\text {bright }}$ decreases $[84,85,90]$. Furthermore, the expression of CD57 is increased on CD56 ${ }^{\mathrm{dim}}$ NK cells from elderly subjects presenting a highly differentiated subset of NK cells. The accumulation of this highly differentiated CD56 ${ }^{\mathrm{dim}} \mathrm{CD} 57+\mathrm{NK}$ cells with aging might explain the functional changes observed with aging i.e. decreased proliferative response to cytokines, preservation of the CD16 mediated cytotoxic capacity [95]. Aging does not affect neither CD16 expression nor functionality. These observations were further extended recently by the findings that CD94 (members of the C-type lectin family) and KLRG1 expressions on NK cells were significantly decreased in elderly subjects [85, 96]. Although, the exact consequence of this decrease is not known it was hypothesized that the decreased expression of these surface markers induces unregulated cell lysis leading to chronic inflammatory conditions. Moreover, the same study revealed the presence of a greater proportion of IFN $\gamma$-positive $\mathrm{CD}^{-} \mathrm{CD} 56^{\text {bright }} \mathrm{NK}$ cells with aging. However, this contrasts with the earlier mentioned studies $[81,86,89,93]$. The difference may originate from the stimulation which was IL-15 versus IL-2, respectively. This may suggest that with aging a shift to a more cytotoxic, cytokine producing and potentially immunomodulatory NK cell phenotype occur as a compensatory mechanism to the decreased proportion of CD56 $6^{\text {bright }}$ NK cells.

Aging also impacts on the kinetics of NK cells [97]. NK cells from elderly have a significantly decreased proliferation and production rate which means with the maintained NK cell number that there is an increased proportion of longliving NK cells which can be related to the increased proportion of CD56 ${ }^{\mathrm{dim}}$ NK cells. The increased expression of CD57 can also suggest that the NK cells of elderly are terminally differentiated cells [81]. These observations are supported by the short telomere length observed in NK cells with aging [98].

Together, although the number of NK cells is increasing with age there is a profound redistribution of NK cell subsets concomitantly to the altered receptor expression and signalling with aging explaining the functional alterations leading either to decreased direct defense against virus-infected and tumor cells or a decreased regulatory activity on other components of the innate immune response ultimately resulting in the decreased modulation of the adaptive immune response. Concomitantly, certain functions, such as cytotoxicity seems to be well preserved in very healthy elderly. However, a lot of work remains to be done to completely understand the role of NK cells in immunosenescence and agerelated diseases.

\section{HOW AGING AFFECTS NKT AND $\gamma \delta$ T CELLS}

NKT cells are an innate $\mathrm{T}$ lymphocyte population that recognizes lipid antigens presented in the context of the CD1d molecule which is found in monocytes, macrophages and DCs [99]. These cells are able to inhibit autopathogenic $\mathrm{T}$ cells while activating pro-inflammatory antigen specific $\mathrm{T}$ cell responses [100]. They are able to secrete both Th1 and Th2 cytokines [101]. They can increase the functions of NK cells [102]. These cells are rapidly recruited from the circulation during acute inflammation and interact with various APCs expressing the CD1d molecule. Recently, it has been shown that NKT cells are able to recruit neutrophils and activate them via their IFN- $\gamma$ secretion [103]. Thus, NKT cells may play an important regulatory role in the acute phase of a microbial aggression by interacting with various APCs via CD1d lipid antigenic presentation and various cytokine secretions.

There are only few reports on NKT cell functioning with aging, mostly murine studies $[67,104,105]$. However it can be hypothesized that the altered activation of APCs via their TLR receptors will create an unfavourable milieu for NKT activation either directly or by their cytokine secretion. Thus, future studies should elucidate how NKT cells are recruited to the site of inflammation, how they are activated and what is their regulatory role on the chronic phase of inflammation in elderly subjects [106].

IL-17 is secreted by the $\gamma \delta \mathrm{T}$ cells, and NK T cells. This cytokine acts indirectly on neutrophil survival through stimulation of the secretion of G-CSF. IL-17 is also released by neutrophils themselves and reinforces their survival and recruitment [15]. How aging affects the $\gamma \delta \mathrm{T}$ cells has not been established to date.

\section{DOES AGING AFFECT THE LINK BETWEEN THE INNATE AND ADAPTIVE IMMUNE SYSTEMS?}

It is becoming more evident that the first line of defense of the organism, the innate immune response is not only a powerful eradicator of foreign invaders, but is also responsible for the activation of the adaptive immune system for long lasting and highly specific immunity by antigen specific, clonally expanded $\mathrm{B}$ and $\mathrm{T}$ lymphocytes. The reduced functioning of both monocytes and DCs with aging will lead to reduced antigen presentation and activation of T cell immune response by these APCs. In addition, neutrophils secrete many molecules which can directly induce DC maturation or the activation of both the innate and the adaptive immune response such as HMG-B1 and alarmins (Fig. 3). It is possible that the reduced neutrophil function with aging will also affect this aspect of their role in immune responses.

Monocytes/Macrophages, additionally to their antigen presentation capacities, are very important to drive the $T$ cells towards a Th1 or Th2 response. Antigen-presenting cells will release a myriad of soluble mediators for $\mathrm{T}$ cell polarization. The type, intensity and duration of releases of these soluble mediators will greatly depend on the capacity of antigen presenting cells to be activated. For instance, the environment in which antigen-presentation occurs is likely to influence the quantity and quality of the message transmitted 
Dysregulation
of innate cells

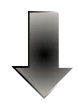

Impact on

adaptive cells

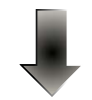

\section{- Pathogen uptake $\longrightarrow \bullet \downarrow$ Antigen delivery by APC}

- Secretion of mediators $\longrightarrow \bullet \downarrow$ Maturation of DC

- Cytokine milieu $\longrightarrow \bullet \downarrow$ APC/T cell/B cell activation and function

- Free radicals $\longrightarrow \cdot \uparrow$ Cell damage

Fig. (3). From innate to adaptive immunity: impact of aging. Effects of the dysregulated innate immune system on essential functions of the adaptive immune system with aging. The change in the milieu in which innate cells circulate will influence their capacity to uptake pathogens and get rid of infection. In the event the innate function are altered (secretion of mediators, free radical production), immune cells from the adaptive arm will initiate their functions under sub-optimal conditions. This impacts on the overall immune response. APC: antigen presenting cells; DC: dendritic cells.

from the membrane (via receptors) to the nucleus, i.e. signalling. Since free radicals are major actors of intracellular signalling, the age-associated pro-inflammatory and prooxidative profile may significantly influence signalling pathways and cross-talks leading to an altered intracellular signalling. Consequently, the induction of the corresponding genes will be significantly altered leading to diminished or inappropriate activity of the cells. This later impacts on $\mathrm{T}$ cell polarization, activity and response.

There is a very efficient network among the various cells participating in the innate immune response aiming to eradicate the invaders, restore the basal situation by resuming the inflammation and ultimately to efficiently activate the adaptive immune response. As we have seen the individual functioning of the innate immune cells is dysregulated with aging either because of cell membrane changes with associated receptor driven signalling pathway alterations or because an age-related pro-inflammatory milieu sustained by cytokines and the oxidative stress [16]. These alterations will induce a disruption in their functioning and in their mutually supporting network resulting ultimately in the altered and inadequate activation of the adaptive immune response.

\section{INNATE IMMUNOSENESCENCE AND AGE- ASSOCIATED PATHOLOGIES}

The apparent disequilibrium between the retention of a relatively reactive innate immune response and the more severely altered adaptive immune response with aging leads to the presence of a low grade inflammatory status commonly present in the elderly and termed inflamm-aging $[107,108]$. Although the cause of this increased basal inflammatory state is certainly multifactorial, it is likely that one of the most important causes is chronic antigenic stimulation. The antigen source can be exogenous, as with persistent viral infections such as CMV [109] and sub-clinical bac- terial infections, or endogenous like the various posttranslationally-modified macromolecules such as DNA or proteins which can be modified by oxidation, acylation or glycosylation. Such altered molecules can stimulate the in nate immune response, particularly macrophages via TLRs, thus contributing to sustaining a pro-inflammatory state. This is measurable in some circumstances as increased circulating levels of IL-6, IL-1 $\beta$ or TNF $\alpha$ (Fig. 2). These modifications may also result in the stimulation of adaptive immune responses, represented in an extreme form by an inverted CD4: CD8 ratio $(<1)$, caused by an overwhelming expansion of CD8+ cells [110,111]. Thus, aging is accompanied by a chronic low-grade inflammatory process. Hence, this may be the price that has to be paid for maintaining immune surveillance against persistent pathogens or endogenous stressors such as cancer cells. All these changes contribute to a decreasingly effective immune environment that seems not to be able to respond appropriately to new antigens such as represented by the continuous emergence of new and chronically existing antigens such as CMV or tumour cells during human lifespan.

Furthermore, an important aspect of the inflammatory response is the production of free radicals which leads to the activation of various signalling cascades resulting in effector functions and apoptosis as well as in the further production of pro-inflammatory cytokines (Fig. 2) [16,112]. These proinflammatory cytokines secreted by the cells of the innate immune response are able in turn also to induce the production of free radicals. The chronic low grade inflammation has been shown in large scale epidemiological studies to be associated with various age-related pathologies cancer, atherosclerosis, Alzheimer's disease [54]. Thus, the deregulation of innate immune response strongly contributes to the agerelated chronic inflammatory processes and associated pathologies and is by no means an inert consequence of the aging process. As such its modulation could be beneficial in the treatment of these diseases. 
Table 1. Phenotype and Function Changes with Aging in Cells of the Innate Immune System

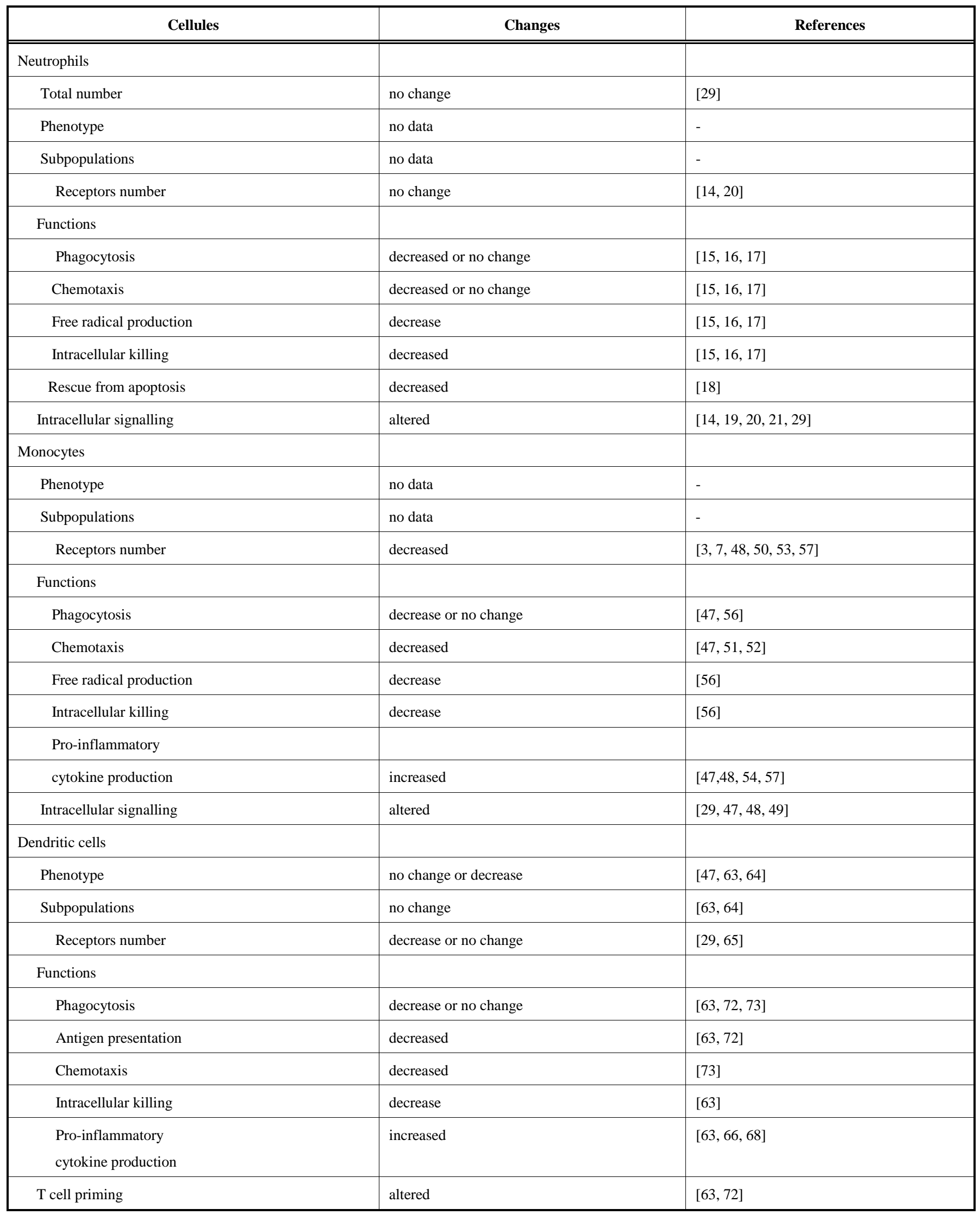


Table 1. Contd.....

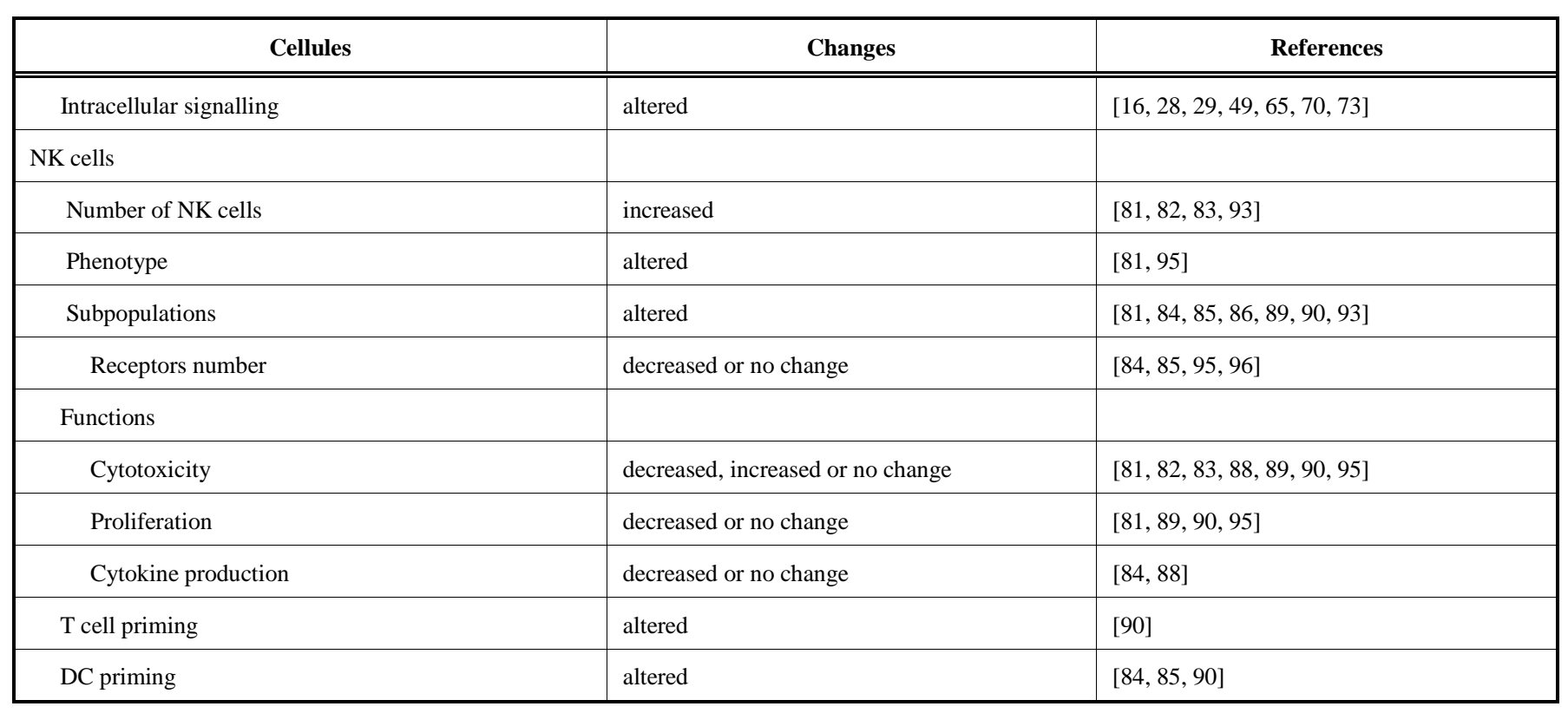

\section{CONCLUSION}

Originally it was thought that immunosenescence was only an alteration of the adaptive immune response. More recently many functional alterations have been demonstrated in various cells of the innate immune response with aging. (Table 1). Importantly, these changes could explain some of the increased age-related morbidity, including not only increased infection rates but also the major age-related pathologies such as cardiovascular disease, dementia and cancer. Moreover, a decline in innate immune function has consequences for the adaptive immune response as these two are closely integrated. This means that if with aging we assist to an alteration of the innate immune response the adaptive immune response will be also altered. Thus, much more research effort should be put on the elucidation of the alterations of the innate immune response in relation to the adaptive immune response to increase the efficacy of the immune system, improve vaccination responses and decrease the occurrence of age-related diseases.

\section{CONFLICT OF INTEREST}

None declared.

\section{ACKNOWLEDGEMENTS}

This work partly supported by grants from the Canadian Institutes of Health Research (CIHR) (No106634, 106701), from the Université de Sherbrooke and from the Research Center on Aging.

\section{REFERENCES}

[1] Caruso C, Buffa S, Candore G, et al. Mechanisms of immunosenescence. Immun Ageing 2009; 6: 10.
[2] Larbi A, Franceschi C, Mazzatti D, Solana R, Wikby A, Pawelec G. Aging of the immune system as a prognostic factor for human longevity. Physiology (Bethesda) 2008; 23: 64-74.

[3] Agarwal S, Busse PJ. Innate and adaptive immunosenescence. Ann Allergy Asthma Immunol 2010; 104(3): 183-90.

[4] Schneider EL. Infectious diseases in the elderly. Ann Intern Med 1983; 98: 395-400.

[5] Biron CA. More things in heaven and earth: defining innate and adaptive immunity. Nat Immunol 2010; 11: 1080-2.

[6] Miller RA. The aging immune system: primer and prospectus. Science 1996; 273: 70-4.

[7] Shaw AC, Joshi S, Greenwood H, Panda A, Lord JM. Aging of the innate system. Curr Opin Immunol 2010; 22: 507-13.

[8] Weiskopf D, Weinberger B, Grubeck-Loebenstein B. The aging of the immune system. Transpl Int 2009; 22: 1041-50.

[9] Biswas SK, Mantovani A. Macrophage plasticity and interaction with lymphocyte subsets: cancer as a paradigm. Nat Immunol 2010; 11(10): 889-96.

[10] Kumar V, Sharma A. Neutrophils: Cinderella of innate immune system. Int Immunopharmacol 2010; 10: 1325-34.

[11] Hume DA, Ross IL, Himes SR, Sasmono RT, Wells CA, Ravasi T. The mononuclear phagocyte system revisited. J Leukoc Biol 2002; 72(4): 621-7.

[12] Borregaard N. Neutrophils, from marrow to microbes. Immunity 2010; 33: 657-70.

[13] Papayannopoulos V, Zychlinsky A. NETs: a new strategy for using old weapons. Trends Immunol 2009; 30: 513-21

[14] Fulop T, Larbi A, Douziech N, et al. Signal transduction and functional changes in neutrophils with aging. Aging Cell 2004; 3: 21726.

[15] Wessels I, Jansen J, Rink L, Uciechowski P. Immunosenescence of polymorphonuclear neutrophils. ScientificWorldJournal 2010; 10: 145-60.

[16] Peters T, Weiss JM, Sindrilaru A, et al. Reactive oxygen intermediate-induced pathomechanisms contribute to immunosenescence, chronic inflammation and autoimmunity. Mech Ageing Dev 2009; 130: 564-87.

[17] Butcher SK, Chahal H, Nayak L, et al. Senescence in innate immune responses: reduced neutrophil phagocytic capacity and CD16 expression in elderly humans. J Leukoc Biol 2001; 70: 8816. 
[18] Fülöp T Jr., Fouquet C, Allaire P, et al. Changes in apoptosis of human polymorphonuclear granulocytes with aging. Mech Ageing Dev 1997; 96: 15-34.

[19] Fülöp T, Fóris G, Leövey A. Age-related changes in cAMP and cGMP levels during phagocytosis in human polymorphonuclear leukocytes. Mech Ageing Dev 1984; 27: 233-7.

[20] Larbi A, Douziech N, Fortin C, Linteau A, Dupuis G, Fulop T Jr. The role of the MAPK pathway alterations in GM-CSF modulated human neutrophil apoptosis with aging. Immun Ageing 2005; 2: 6.

[21] Fortin CF, McDonald PP, Lesur O, Fülöp T Jr. Aging and neutrophils: there is still much to do. Rejuvenation Res 2008; 11: 873-82.

[22] Janeway CA Jr. Approaching the asymptote? Evolution and revolution in immunology. Cold Spring Harb Symp Quant Biol 1989; 54 (Pt 1): 1-13.

[23] Wu S, Metcalf JP, Wu W. Innate immune response to influenza virus. Curr Opin Infect Dis 2011; 24: 235-40.

[24] Kawai T, Akira S. The role of pattern-recognition receptors in innate immunity: update on Toll-like receptors. Nat Immunol 2010; 11: 373-84.

[25] Kawai T, Akira S. Antiviral signaling through pattern recognition receptors. J Biochem 2007; 141: 137-45.

[26] Casanova JL, Abel L, Quintana-Murci L. Human TLRs and IL-1Rs in host defense: natural insights from evolutionary, epidemiological, and clinical genetics. Annu Rev Immunol 2011; 29: 447-91.

[27] Ostuni R, Zanoni I, Granucci F. Deciphering the complexity of Toll-like receptor signaling. Cell Mol Life Sci 2010; 67(24): 410934.

[28] Kawai T, Akira S. Signaling to NF-kappaB by Toll-like receptors. Trends Mol Med 2007; 13: 460-9.

[29] Shaw AC, Panda A, Joshi SR, Qian F, Allore HG, Montgomery RR. Dysregulation of human Toll-like receptor function in aging. Ageing Res Rev 2011; 10: 346-53.

[30] Pichlmair A, Schulz O, Tan CP, et al. RIG-I-mediated antiviral responses to single-stranded RNA bearing 5'-phosphates. Science 2006; 314: 997-1001.

[31] Franchi L, Eigenbrod T, Muñoz-Planillo R, Nuñez G. The inflammasome: a caspase-1-activation platform that regulates immune responses and disease pathogenesis. Nat Immunol 2009; 10: 241-7.

[32] Khare S, Luc N, Dorfleutner A, Stehlik C. Inflammasomes and their activation. Crit Rev Immunol 2010; 30: 463-87.

[33] Brinkmann V, Reichard U, Goosmann C, et al. Neutrophil extracellular traps kill bacteria. Science 2004; 303: 1532-5.

[34] Metzler KD, Fuchs TA, Nauseef WM, et al. Myeloperoxidase is required for neutrophil extracellular trap formation: implications for innate immunity. Blood 2011; 117: 953-9.

[35] Hakkim A, Fürnrohr BG, Amann K, et al. Impairment of neutrophil extracellular trap degradation is associated with lupus nephritis. Proc Natl Acad Sci USA 2010 25; 107: 9813-8.

[36] Minet-Quinard R, Farges MC, Thivat E, et al. Neutrophils are immune cells preferentially targeted by retinoic acid in elderly subjects. Immun Ageing 2010; 7: 10.

[37] Geissmann F, Manz MG, Jung S, Sieweke MH, Merad M, Ley K. Development of monocytes, macrophages, and dendritic cells. Science 2010; 327: 656-61.

[38] Cros J, Cagnard N, Woollard K, et al. Human CD14dim monocytes patrol and sense nucleic acids and viruses via TLR7 and TLR8 receptors. Immunity 2010; 33: 375-86.

[39] Geissmann F, Gordon S, Hume DA, Mowat AM, Randolph GJ. Unravelling mononuclear phagocyte heterogeneity. Nat Rev Immunol 2010; 10: 453-60.

[40] Mege JL, Mehraj V, Capo C. Macrophage polarization and bacterial infections. Curr Opin Infect Dis 2011; 24: 230-4.

[41] Chertov O, Ueda $\mathrm{H}, \mathrm{Xu}$ LL, et al. Identification of human neutrophil-derived cathepsin G and azurocidin/CAP37 as chemoattractants for mononuclear cells and neutrophils. J Exp Med 1997; 186: 739-47.

[42] Bennouna S, Bliss SK, Curiel TJ, Denkers EY. Cross-talk in the innate immune system: neutrophils instruct recruitment and activation of dendritic cells during microbial infection. J Immunol 2003; $171: 6052-8$

[43] Tsuda Y, Takahashi H, Kobayashi M, Hanafusa T, Herndon DN, Suzuki F. Three different neutrophil subsets exhibited in mice with different susceptibilities to infection by methicillin-resistant Staphylococcus aureus. Immunity 2004; 21: 215-26

[44] Ethuin F, Gérard B, Benna JE, et al. Human neutrophils produce interferon gamma upon stimulation by interleukin-12. Lab Invest 2004; 84: 1363-71.

[45] Plackett TP, Boehmer ED, Faunce DE, Kovacs EJ. Aging and innate immune cells. J Leukoc Biol 2004; 76: 291-9.

[46] Hajishengallis G. Too old to fight? Aging and its toll on innate immunity. Mol Oral Microbiol 2010; 25: 25-37.

[47] Aw D, Silva AB, Palmer DB. Immunosenescence: emerging challenges for an ageing population. Immunology 2007; 120: 435-46.

[48] Panda A, Arjona A, Sapey E, et al. Human innate immunosenescence: causes and consequences for immunity in old age. Trends Immunol 2009; 30: 325-33.

[49] Crétel E, Veen I, Pierres A, Bongrand P, Gavazzi G. Immunosenescence and infections, myth or reality? Med Mal Infect 2010; 40: 307-18.

[50] Renshaw M, Rockwell J, Engleman C, Gewirtz A, Katz J, Sambhara S. Cutting edge: impaired Toll-like receptor expression and function in aging. J Immunol 2002; 169: 4697-701.

[51] Plowden J, Renshaw-Hoelscher M, Engleman C, Katz J, Sambhara S. Innate immunity in aging: impact on macrophage function. Aging Cell 2004; 3: 161-7.

[52] Ashcroft GS, Horan MA, Ferguson MW. Aging alters the inflammatory and endothelial cell adhesion molecule profiles during human cutaneous wound healing. Lab Invest 1998; 78: 47-58.

[53] van Duin D, Allore HG, Mohanty S, et al. Prevaccine determination of the expression of costimulatory B7 molecules in activated monocytes predicts influenza vaccine responses in young and older adults. J Infect Dis 2007; 195: 1590-7.

[54] Franceschi C, Capri M, Monti D, et al. Inflammaging and antiinflammaging: a systemic perspective on aging and longevity emerged from studies in humans. Mech Ageing Dev 2007; 128: 92105.

[55] Wu D, Hayek MG, Meydani S. Vitamin E and macrophage cyclooxygenase regulation in the aged. J Nutr 2001; 131: 382S-8.

[56] Gomez CR, Nomellini V, Faunce DE, Kovacs EJ. Innate immunity and aging. Exp Gerontol 2008; 43: 718-28.

[57] Kong KF, Delroux K, Wang X, et al. Dysregulation of TLR3 impairs the innate immune response to West Nile virus in the elderly. J Virol 2008; 82: 7613-23.

[58] Liu YJ. Dendritic cell subsets and lineages, and their functions in innate and adaptive immunity. Cell 2001;106(3): 259-62.

[59] Romagnani C, Della Chiesa M, Kohler S, et al. Activation of human NK cells by plasmacytoid dendritic cells and its modulation by CD4+ T helper cells and CD4+ CD25hi T regulatory cells. Eur J Immunol 2005; 35: 2452-8.

[60] Ferlazzo G, Pack M, Thomas D, et al. Distinct roles of IL-12 and IL-15 in human natural killer cell activation by dendritic cells from secondary lymphoid organs. Proc Natl Acad Sci USA 2004; 101: 16606-11.

[61] Lande R, Gilliet M. Plasmacytoid dendritic cells: key players in the initiation and regulation of immune responses. Ann N Y Acad Sci 2010; 1183: 89-103.

[62] Lanzavecchia A, Sallusto F. Regulation of T cell immunity by dendritic cells. Cell 2001; 106: 263-6.

[63] Agrawal A, Gupta S. Impact of aging on dendritic cell functions in humans. Ageing Res Rev 2011; 10: 336-45.

[64] Jing Y, Shaheen E, Drake RR, Chen N, Gravenstein S, Deng Y Aging is associated with a numerical and functional decline in plasmacytoid dendritic cells, whereas myeloid dendritic cells are relatively unaltered in human peripheral blood. Hum Immunol 2009; 70: 777-84.

[65] Stout-Delgado HW, Yang X, Walker WE, Tesar BM, Goldstein DR. Aging impairs IFN regulatory factor 7 up-regulation in plasmacytoid dendritic cells during TLR9 activation. J Immunol 2008; 181: 6747-56.

[66] Agrawal A, Agrawal S, Tay J, Gupta S. Biology of dendritic cells in aging. J Clin Immunol 2008; 28: 14-20.

[67] Stout-Delgado HW, Du W, Shirali AC, Booth CJ, Goldstein DR. Aging promotes neutrophil-induced mortality by augmenting IL-17 production during viral infection. Cell Host Microbe 2009; 6: 44656. 
[68] Leng J, Goldstein DR. Impact of aging on viral infections. Microbes Infect 2010; 12: 1120-4.

[69] Katz JM, Plowden J, Renshaw-Hoelscher M, Lu X, Tumpey TM, Sambhara S. Immunity to influenza: the challenges of protecting an aging population. Immunol Res 2004; 29: 113-24.

[70] Panda A, Qian F, Mohanty S, et al. Age-associated decrease in TLR function in primary human dendritic cells predicts influenza vaccine response. J Immunol 2010; 184: 2518-27.

[71] Schmitz N, Kurrer M, Bachmann MF, Kopf M. Interleukin-1 is responsible for acute lung immunopathology but increases survival of respiratory influenza virus infection. J Virol 2005; 79: 6441-8.

[72] Della Bella S, Bierti L, Presicce P, et al. Peripheral blood dendritic cells and monocytes are differently regulated in the elderly. Clin Immunol 2007; 122: 220-8.

[73] Agrawal A, Agrawal S, Cao JN, Su H, Osann K, Gupta S. Altered innate immune functioning of dendritic cells in elderly humans: a role of phosphoinositide 3-kinase-signaling pathway. J Immunol 2007; 178: 6912-22.

[74] Altfeld M, Fadda L, Frleta D, Bhardwaj N. DCs and NK cells: critical effectors in the immune response to HIV-1. Nat Rev Immunol 2011; 11: 176-86.

[75] Biron CA, Nguyen KB, Pien GC, Cousens LP, Salazar-Mather TP. Natural killer cells in antiviral defense: function and regulation by innate cytokines. Annu Rev Immunol 1999; 17: 189-220.

[76] Fernandez NC, Lozier A, Flament C, et al. Dendritic cells directly trigger NK cell functions: cross-talk relevant in innate anti-tumor immune responses in vivo. Nat Med 1999; 5: 405-11.

[77] Mailliard RB, Son YI, Redlinger R, et al. Dendritic cells mediate NK cell help for Th1 and CTL responses: two-signal requirement for the induction of NK cell helper function. J Immunol 2003; 171: 2366-73.

[78] Wendt K, Wilk E, Buyny S, Buer J, Schmidt RE, Jacobs R. Gene and protein characteristics reflect functional diversity of CD56dim and CD56bright NK cells. J Leukoc Biol 2006; 80: 1529-41.

[79] Parham P. Taking license with natural killer cell maturation and repertoire development. Immunol Rev 2006; 214: 155-60.

[80] Watzl C, Long EO. Signal transduction during activation and inhibition of natural killer cells. Curr Protoc Immunol 2010; Chapter 11: Unit 11.9B.

[81] Borrego F, Alonso MC, Galiani MD, et al. NK phenotypic markers and IL2 response in NK cells from elderly people. Exp Gerontol 1999; 34: 253-65.

[82] Ligthart GJ, Schuit HR, Hijmans W. Natural killer cell function is not diminished in the healthy aged and is proportional to the number of NK cells in the peripheral blood. Immunology 1989; 68: 396-402.

[83] Sansoni P, Cossarizza A, Brianti V, et al. Lymphocyte subsets and natural killer cell activity in healthy old people and centenarians. Blood 1993; 82: 2767-73.

[84] Le Garff-Tavernier M, Béziat V, Decocq J, et al. Human NK cells display major phenotypic and functional changes over the life span. Aging Cell 2010; 9: 527-35.

[85] Almeida-Oliveira A, Smith-Carvalho M, Porto LC, et al. Agerelated changes in natural killer cell receptors from childhood through old age. Hum Immunol 2011; 72: 319-29.

[86] Ogata K, An E, Shioi Y, et al. Association between natural killer cell activity and infection in immunologically normal elderly people. Clin Exp Immunol 2001; 124: 392-7.

[87] Ogata K, Yokose N, Tamura H, et al. Natural killer cells in the late decades of human life. Clin Immunol Immunopathol 1997; 84: 269-75.

[88] Mocchegiani E, Giacconi R, Cipriano C, Malavolta M. NK and NKT cells in aging and longevity: role of zinc and metallothioneins. J Clin Immunol 2009; 29: 416-25.

[89] Mocchegiani E, Malavolta M. NK and NKT cell functions in immunosenescence. Aging Cell 2004; 3: 177-84

[90] Gayoso I, Sanchez-Correa B, Campos C, et al. Immunosenescence of human natural killer cells. J Innate Immun 2011; 3: 337-43.

[91] Arnon TI, Markel G, Mandelboim O. Tumor and viral recognition by natural killer cells receptors. Semin Cancer Biol 2006; 16: 34858.
[92] Arnon TI, Achdout H, Levi O, et al. Inhibition of the NKp30 activating receptor by pp65 of human cytomegalovirus. Nat Immunol 2005; 6: 515-23.

[93] Krishnaraj R. Senescence and cytokines modulate the NK cell expression. Mech Ageing Dev 1997; 96: 89-101.

[94] Vallejo AN, Mueller RG, Hamel DL Jr, et al. Expansions of NKlike $\alpha \beta T$ cells with chronologic aging: novel lymphocyte effectors that compensate for functional deficits of conventional NK cells and T cells. Ageing Res Rev 2011; 10: 354-61.

[95] Lutz CT, Moore MB, Bradley S, Shelton BJ, Lutgendorf SK. Reciprocal age related change in natural killer cell receptors for MHC class I. Mech Ageing Dev 2005; 126: 722-31.

[96] Hayhoe RP, Henson SM, Akbar AN, Palmer DB. Variation of human natural killer cell phenotypes with age: identification of a unique KLRG1-negative subset. Hum Immunol 2010; 71: 676-81.

[97] Zhang Y, Wallace DL, de Lara CM, et al. In vivo kinetics of human natural killer cells: the effects of ageing and acute and chronic viral infection. Immunology 2007; 121: 258-65.

[98] Mariani E, Meneghetti A, Formentini I, et al. Telomere length and telomerase activity: effect of ageing on human NK cells. Mech Ageing Dev 2003; 124: 403-8.

[99] Fox L, Hegde S, Gumperz JE. Natural killer T cells: innate lymphocytes positioned as a bridge between acute and chronic inflammation?. Microbes Infect 2010; 12: 1125-33.

[100] Cerundolo V, Silk JD, Masri SH, Salio M. Harnessing invariant NKT cells in vaccination strategies. Nat Rev Immunol 2009; 9: 2838.

[101] Hegde S, Fox L, Wang X, Gumperz JE. Autoreactive natural killer $\mathrm{T}$ cells: promoting immune protection and immune tolerance through varied interactions with myeloid antigen-presenting cells. Immunology 2010; 130: 471-83.

[102] Eberl G, MacDonald HR. Selective induction of NK cell proliferation and cytotoxicity by activated NKT cells. Eur J Immunol 2000; 30: 985-92.

[103] Nakamatsu M, Yamamoto N, Hatta M, et al. Role of interferongamma in Valpha14+ natural killer T cell-mediated host defense against Streptococcus pneumoniae infection in murine lungs. Microbes Infect 2007; 9: 364-74.

[104] Faunce DE, Palmer JL, Paskowicz KK, Witte PL, Kovacs EJ. CD1d-restricted NKT cells contribute to the age-associated decline of T cell immunity. J Immunol 2005; 175: 3102-9.

[105] Inui T, Nakagawa R, Ohkura S, et al. Age-associated augmentation of the synthetic ligand- mediated function of mouse NK1.1 ag(+) T cells: their cytokine production and hepatotoxicity in vivo and in vitro. J Immunol 2002; 169: 6127-32.

[106] Solana R, Mariani E. NK and NK/T cells in human senescence. Vaccine 2000; 18: 1613-20.

[107] Franceschi C, Bonafè M, Valensin S, et al. Inflamm-aging. An evolutionary perspective on immunosenescence. Ann N Y Acad Sci 2000; 908: 244-54.

[108] Candore G, Caruso C, Jirillo E, Magrone T, Vasto S. Low grade inflammation as a common pathogenetic denominator in agerelated diseases: novel drug targets for anti-ageing strategies and successful ageing achievement. Curr Pharm Des 2010; 16: 584-96.

[109] Pawelec G, Derhovanessian E, Larbi A, Strindhall J, Wikby A. Cytomegalovirus and human immunosenescence. Rev Med Virol 2009; 19: 47-56.

[110] Wikby A, Johansson B, Olsson J, Löfgren S, Nilsson BO, Ferguson F. Expansions of peripheral blood CD8 T-lymphocyte subpopulations and an association with cytomegalovirus seropositivity in the elderly: the Swedish NONA immune study. Exp Gerontol 2002; 37: 445-53.

[111] Olsson J, Wikby A, Johansson B, Löfgren S, Nilsson BO, Ferguson FG. Age-related change in peripheral blood T-lymphocyte subpopulations and cytomegalovirus infection in the very old: the Swedish longitudinal OCTO immune study. Mech Ageing Dev 2000; 121: 187-201.

[112] Khansari N, Shakiba Y, Mahmoudi M. Chronic inflammation and oxidative stress as a major cause of age-related diseases and cancer. Recent Pat Inflamm Allergy Drug Discov 2009; 3: 73-80. 